\title{
Lipid Resuscitation Reverses Multidrug-Induced Cardiogenic Shock
}

\author{
Israel Rubinstein • Brian Zider • Guy Weinberg
}

Published online: 27 August 2011

(C) American College of Medical Toxicology 2011

To the Editor:

We read with interest the recent case report by Stellpflug describing the eventual recovery of a 30 -year-old patient with severe cardiovascular instability due to mixed diltiazem, metoprolol, and amiodarone overdose. However, we have strong reservations about their conclusion that, "The treatment combination........ was successful for this patient in reversing severe drug-induced cardiogenic shock." The patient received high-dose insulin for $85 \mathrm{~min}$ which the authors state, "...did not result in clinical improvement...." Then after intravenous lipid emulsion (ILE 20\%) therapy, the authors report, “.... was associated with rapid improvement." The chronology suggests to us that ILE saved the patient's life after prolonged, ineffective high-dose insulin therapy. This conforms with many other reports of successful resuscitation with ILE in patients that did not respond to conventional (e.g., pressor) treatment. We believe the authors' own comments strongly contradict their conclusion. Everyone would agree, we expect, that the report provides a rationale for further head-to-head laboratory comparison of ILE with high-dose insulin in treatment of mixed drug overdose and particularly calcium channel blocker-related cardiogenic shock.

Dr. Weinberg was awarded with the United States patent 7261903 B1 "Lipid emulsion in the treatment of systemic poisoning." The noncommercial website www.lipidregistry. org is intended for the purpose of case documentation. Neither salary nor support is derived from this website.

I. Rubinstein

Department of Medicine, University of Illinois at Chicago,

Chicago, IL, USA

B. Zider $(\bowtie) \cdot$ G. Weinberg

Department of Anesthesiology, University of Illinois at Chicago,

Chicago, IL, USA

e-mail: bzider2@uic.edu 